\title{
Provocation and prediction of visual peripersonal neglect-like symptoms in preoperative planning and during awake brain surgery
}

\author{
Viktória Tamás ${ }^{1}$ • Gabriella Sebestyén ${ }^{1}$ - Szilvia Anett Nagy ${ }^{2,3,4,5}$ • Péter Zsolt Horváth ${ }^{1}$. Ákos Mérei ${ }^{6}$. \\ Francesco Tomaiuolo ${ }^{7}$ • Giovanni Raffa ${ }^{8}$ - Antonino Francesco Germanó ${ }^{8}$ • András Büki ${ }^{1,9}$
}

Received: 18 February 2021 / Accepted: 18 March 2021 / Published online: 5 April 2021

(C) The Author(s) 2021

\begin{abstract}
Neglect is a severe neuropsychological/neurological deficit that usually develops due to lesions of the posterior inferior parietal area of the right hemisphere and is characterized by a lack of attention to the left side. Our case is a proven right-handed, 30-yearold female patient with a low-grade glioma, which was located in the temporo-opercular region and also in the superior temporal gyrus of the right hemisphere. Upon presurgical planning, the motor, language, and visuospatial functions were mapped. In order to achieve this, the protocol for routine magnetic resonance imaging and navigated transcranial magnetic stimulation has been expanded, accordingly.
\end{abstract}

Keywords awake brain surgery $\cdot$ brain tumor $\cdot$ visual neglect $\cdot$ nTMS

\section{Introduction}

Recent clinical studies suggest the important role of supramaximal safe tumor resection in increasing life expectancy of patients with low-grade gliomas. During extended resective surgery, it is of utmost importance to spare functionally eloquent areas. This goal is best achieved by awake surgery, aided by intraoperative neuromonitoring and cognitive assessment. Neglect is a severe neuropsychological/ neurological deficit characterized by the lack of attention for the left side of the space. It develops as a result of a lesion at the posterior section of the inferior-parietal region mostly of the right hemisphere $[1,6,8]$.

This article is part of the Topical Collection on Brain Tumors

Viktória Tamás

tamas.viktoria@pte.hu

1 Department of Neurosurgery, Medical School, University of Pécs, Pécs, Hungary

2 MTA-PTE Clinical Neuroscience MR Research Group, Pécs, Hungary

3 János Szentágothai Research Centre, University of Pécs, Pécs, Hungary

4 Pécs Diagnostic Center, Pécs, Hungary

\section{Case report}

\section{Subject}

A 30-year-old right-handed female has been diagnosed with a suspected low-grade glioma of the temporo-opercular region and superior temporal gyrus of the right hemisphere, affecting both cortical and subcortical areas. The patient has provided an informed consent acknowledging purported defect in the visual field.

\section{Mapping of the eloquent areas}

Preoperative imaging workup included structural magnetic resonance imaging (MRI), diffusion tensor imaging (DTI) to

5 Department of Laboratory Medicine, Medical School, University of Pécs, Pécs, Hungary

6 Department of Anesthesiology and Intensive Therapy, Medical School, University of Pécs, Pécs, Hungary

7 Department of Clinical and Experimental Medicine, University of Messina, Messina, Italy

8 Department of Biomedical, Dental, Morphological and Functional Imaging Sciences, University of Messina, Messina, Italy

9 Neurotrauma Research Group, Szentágothai Research Centre, University of Pécs, Pécs, Hungary 
Fig. 1 Line bisection judgment task, referred to as the landmark task to pre- and intraoperative mapping

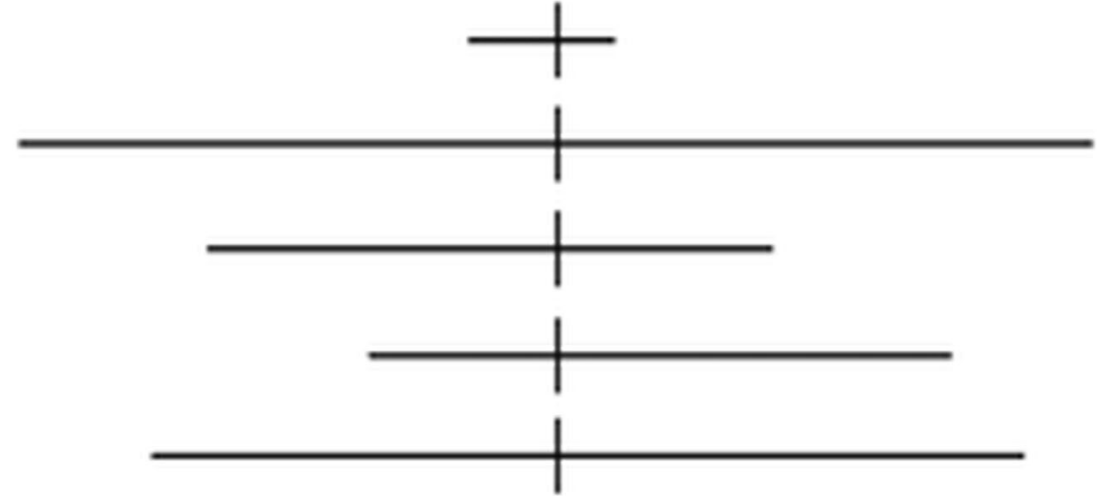

Cortical mapping was performed with nTMS using evaluate white matter tracts and functional MRI (fMRI) to Navigated Brain Stimulation system combined with the
Fig.2 a Simultaneous left-sided dominant Wernicke activation and right-sided subdominant activity at the margin of the mass lesion. b Tractrogram of fiber network of FLS. c Tractrogram of fiber network representing the pyramidal tract. d Tractrogram of fiber network representing the tract system projected from the nTMS spots. e Errors in line bisection test during rTMS stimulation. Neglect specific errors are marked with yellow; non-neglect specific errors are marked with orange, delayed reaction times are marked with green

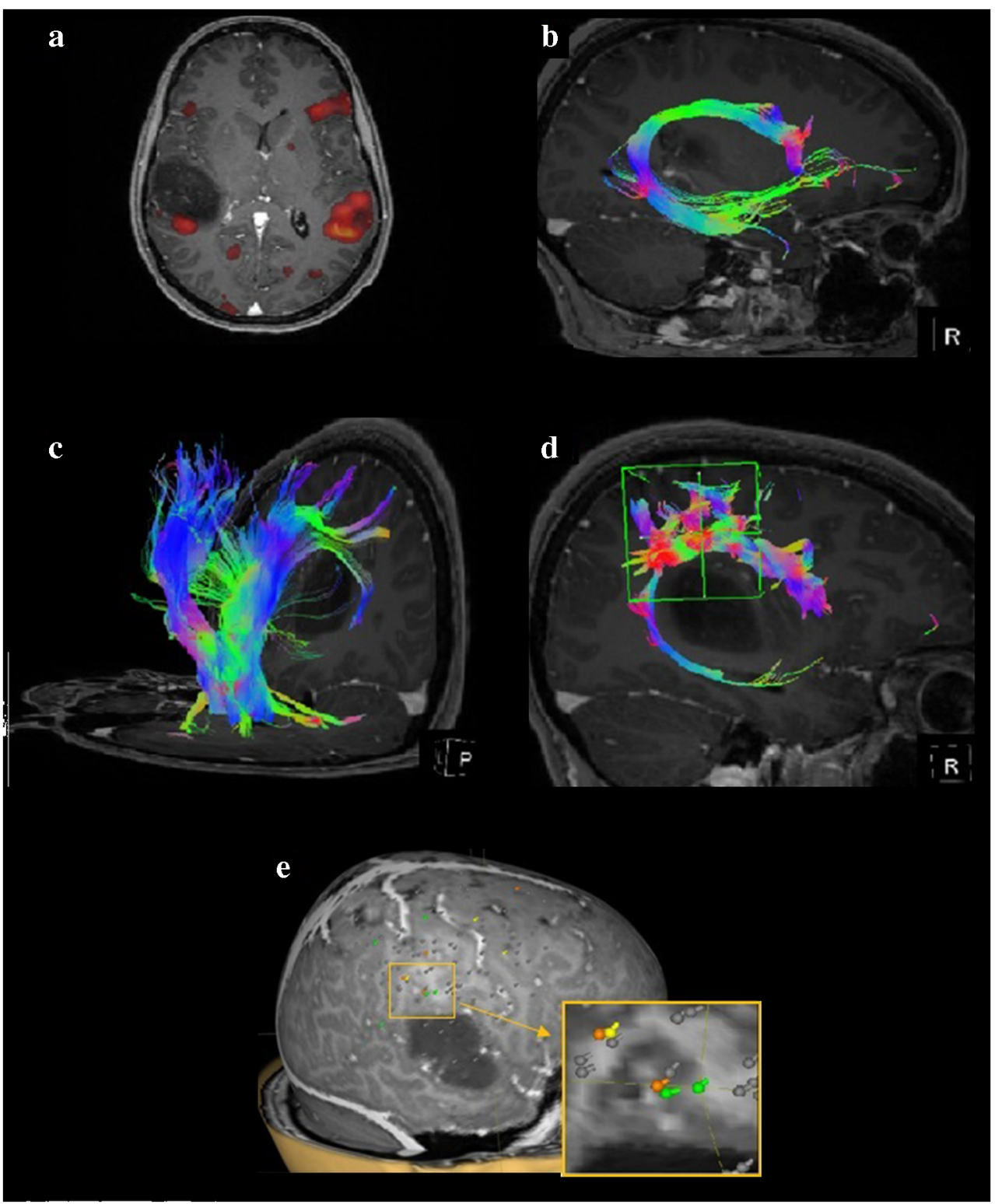


Table 1 Parameters of neglect and non-neglect specified errors

\begin{tabular}{lllll}
\hline $\begin{array}{l}\text { Total line length } \\
(\mathrm{mm})\end{array}$ & $\begin{array}{l}\text { Left side } \\
(\mathrm{mm})\end{array}$ & $\begin{array}{l}\text { Right side } \\
(\mathrm{mm})\end{array}$ & Error & $\begin{array}{l}\text { Neglect (yes/ } \\
\text { no })\end{array}$ \\
\hline 196 & 106 & 90 & Delay Yes \\
159 & 82 & 77 & Equal Yes \\
196 & 92 & 104 & Equal No \\
106 & 50 & 56 & Equal No \\
\hline
\end{tabular}

NEXSPEECH® module to locate the motor cortex, areas of language dominance, and those regions susceptible to neglect.

Although the patient was right-handed, language testing was necessary because the tumor was located in a Wernickehomologue area, which (in a minor region) showed activation during comprehension of speech targeted fMRI testing. Mapping was performed on both hemispheres according to the previously published protocol [5] using trains consisting of $5 \mathrm{~Hz} / 5$ pulses, while the patient was performing an object naming test.

The examination of visuospatial capabilities was especially important because the tumor affected the neglect circlealthough a less sensitive and less extensive region of it, while, in general, it was close to those structures which were widely associated with severe neglect syndrome [7].
During testing for neglect, 44 stimulation target points on the parietal region of the right hemisphere were defined, where a total of 140 stimuli were delivered while the patient was asked to evaluate the length of 80 differently midtransected line ('equal'; 'left' = left is longer; 'right' = right is longer). The pictures have been presented randomly. To aid the inhibitory effect a train of $10 \mathrm{~Hz} / 5$ pulses was used, with $100 \%$ of resting motor threshold.

\section{Visual and verbal stimuli}

Pre- and intraoperative mapping of neglect-eloquent areas were performed by a line bisection judgment task, referred to as the landmark task according to descriptions [2-4].

Our own image library was built by the adaptation of NBS system. Horizontal black lines were split with a vertical line on a white background (Fig. 1).

Wrong judgment of length or the lack of answer were considered errors (neglect and non-neglect specific). Neglectspecific errors were categorized as follows: (1) longer left lines were named as equal; (2) longer left lines were named as longer right lines; (3) equal length lines were named as longer right. Non-neglect specific errors were categorized similarly: (1) longer right lines were named as equal; (2)
Fig. 3 a, b T1-weighted contrastenhanced MRI scan at preoperative phase demonstrate typical appearance of a low-grade glioma without any accumulation of the contrast medium. c, d T1weighted contrast-free follow-up MRI scan $24 \mathrm{~h}$ after surgery revealed gross total removal of the tumor with thin hyperintense line indicative of minimal blood accumulation in the tumor bed

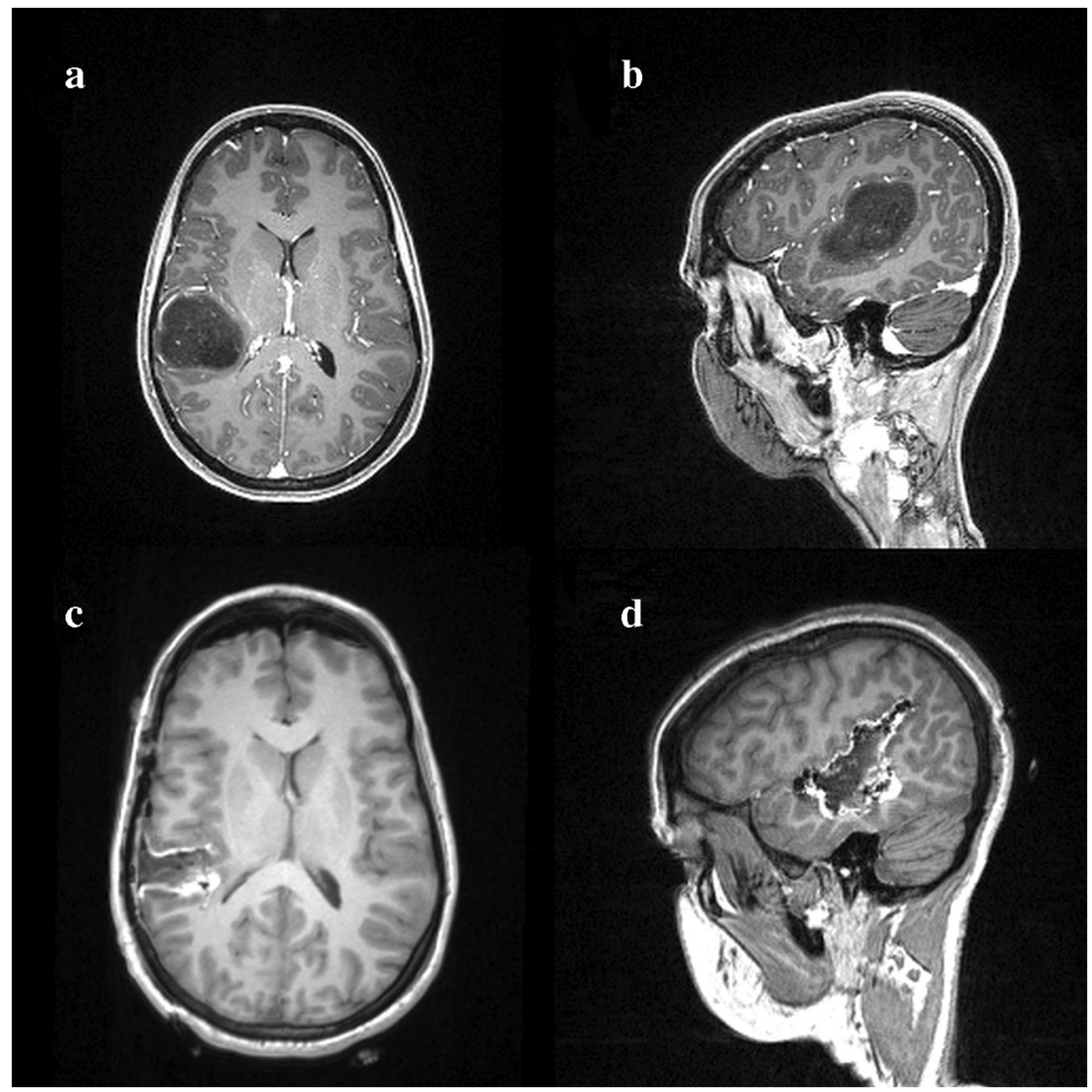


Table 2 Results of psychological tests

\begin{tabular}{|c|c|c|c|}
\hline & Preoperative assessment & Postoperative assessment & Cut-off scores \\
\hline RAVLT1 & 7 & 6 & 6.7 \\
\hline RAVLT2 & 9 & 9 & 9.9 \\
\hline RAVLT3 & 9 & 10 & 11.4 \\
\hline RAVLT4 & 11 & 12 & 12.2 \\
\hline RAVLT5 & 12 & 11 & 12.7 \\
\hline RAVLTB & 4 & 3 & 6.5 \\
\hline RAVLT6-immediate recall & 11 & 8 & 11.2 \\
\hline RAVLT7—delayed recall & 8 & 10 & 11.1 \\
\hline RAVLT—recognition (correct answers) & 15 (+ 9 false intruses) & 13 (+ 0 false intruses) & 14.2 \\
\hline Five-point test (correct patterns) & 13 & 16 & $>10$ \\
\hline Five point test — number of perseverations & 0 & 0 & $<2$ \\
\hline Verbal fluency task — phonological part (FAS)—SUM & 38 & 40 & $\geq 30$ \\
\hline Verbal fluency task — semantic part (animal, vehicle, vegetable)—SUM & 52 & 51 & $\geq 50$ \\
\hline Pieron $(\mathrm{T} \%)$ & 90,9 & 87,3 & 98,9 \\
\hline Pieron $(\mathrm{N})$ & 287 & 269 & 345 \\
\hline Digit span-forward & 5 & 6 & $>5$ \\
\hline Digit span-backward & 3 & 3 & $>4$ \\
\hline TMT A (s) & 46 & 48 & 24.40 \\
\hline TMT B (s) & 50 & 77 & 50.68 \\
\hline FAB & 16 & 18 & 12 \\
\hline Clock drawing test & 10 & 10 & 9 \\
\hline Rey-Osterrieth complex figure test-copying part & 35 & 27 & $\leq 32$ \\
\hline Rey-Osterrieth complex figure test_-immediate recall & 17,5 & 17,5 & $\leq 22$ \\
\hline DWNB — picture naming / $W$ value & 549 & 561 & 540 \\
\hline DWNB — finger tapping/dominant $W$ value & 512 (normal) & 504 (normal) & 508 \\
\hline DWNB — finger tapping/non-dominant/ $W$ value & 515 (normal) & 514 (normal) & 508 \\
\hline DWNB — strength of grip/dominant $/ W$ value & 525 (normal) & 523 (normal) & 530 \\
\hline DWNB - strength of grip/non-dominant $/ W$ value & 524 (normal) & 523 (normal) & 528 \\
\hline Beck anxiety inventory & 5 & 23 & $>21$ \\
\hline Beck depression inventory & 3 & 7 & $>4$ \\
\hline SF-12 & 50 & 27 & $<48$ \\
\hline MD Anderson symptom inventory & 10 & 63 & $>14$ \\
\hline
\end{tabular}

longer right lines were named as longer left lines; (3) equal length lines were named as longer left.

\section{Neuropsychological evaluation}

Mapping of neurocognitive and other psychological functions was performed 2 days prior to, as well as 4 and 5 days after surgery.

A variety of neuropsychological tests were performed for evaluating global neurocognitive functioning. (See Supplementary mat. 1).

To assess visuospatial attention abilities and to detect neglect symptoms, line bisection test (visuospatial attention) and Bells Test (visuospatial attention) were included in our battery. These tests have been performed multiple times following surgery to monitor peripersonal visual neglect symptoms more closely.

\section{Results}

\section{Preoperative phase}

On fMRI, a right-sided subdominant activity appeared by the posterior-inferior margin of the lesion, besides the left-sided dominant Wernicke activation, which was not confirmed with nTMS (Fig. 2a). 

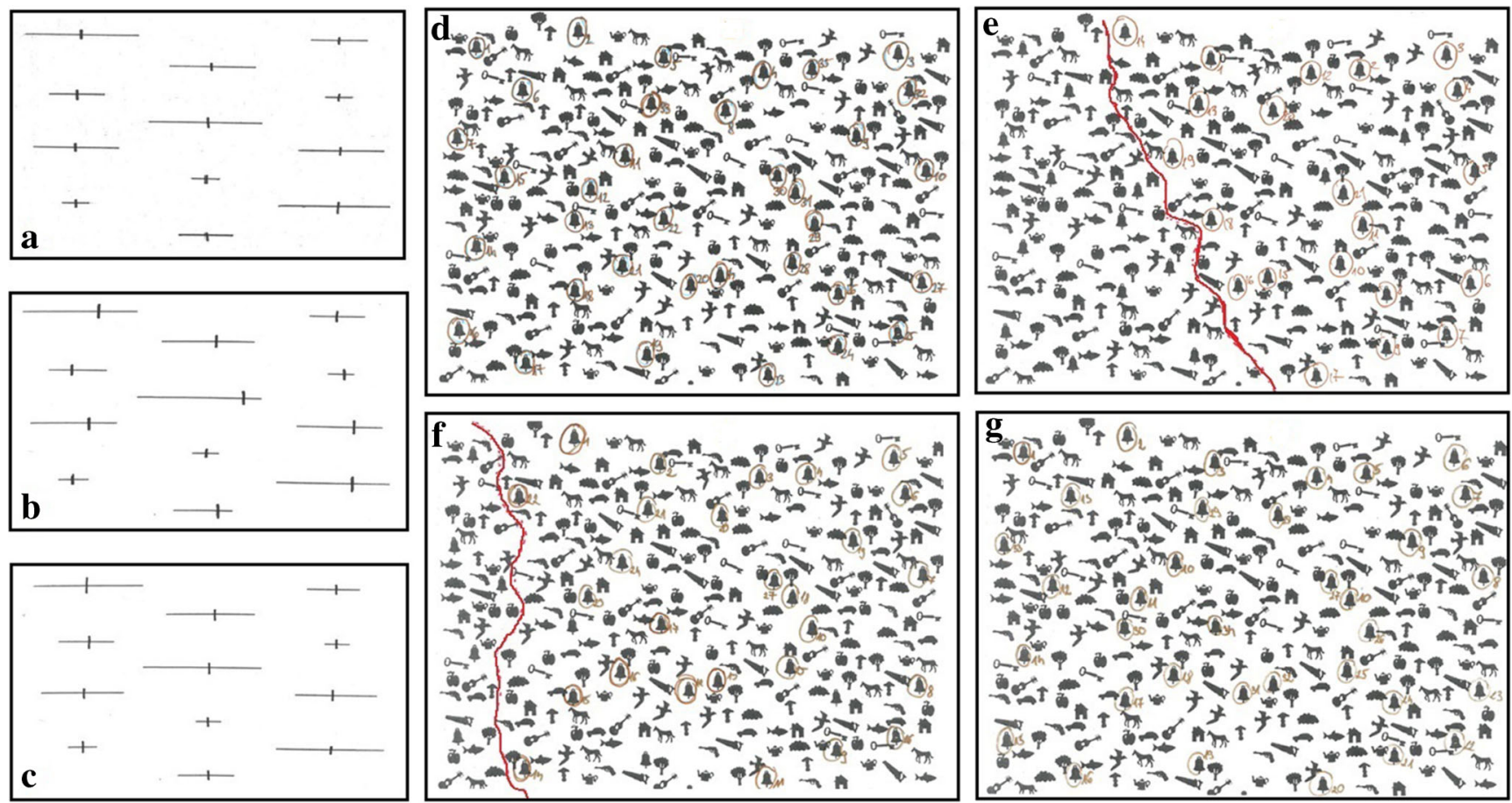

Fig. 4 The alteration in visuospatial attention functions (the appearance of neglect-like symptoms). a Line bisection test—preoperative phase. $\mathbf{b}, \mathbf{c}$

Superior longitudinal fasciculus (FLS) and pyramidal tracts were intact by using DTI and AMRI - a finding confirmed by nTMS examination, too (Fig. 2b-d). A cluster of neglect-like and not neglect-specific spots (4 targets) were identified at the inferior parietal region (Table 1; Fig. 2e). These spots were used for tractographic analysis and for navigation during surgery. Furthermore, activation of other areas (6 instances, e.g., frontal lobe) was recognized, although more scattered than at previously mentioned areas.

Significant neurological and neuropsychological disorder, which refers to contraindication in relationship with the examinations, could not be detected at the patient. Neglect-like symptoms have not been observed and motor functions seemed intact.

\section{Intraoperative phase}

The asleep-awake-asleep operation was planned based on tractography and navigational MRI sequences. During the operation, frameless navigation-guided temporal craniotomy was performed; next functional areas were identified by bipolar fork probe using continuous cortical and subcortical stimulation. During intraoperative stimulation, we were not able to provoke speech error at the location (posterior portion of the superior temporal gyrus) according to nTMS results.

Initially, we barely found symptoms of neglect (1\%) during intraoperative cortical mapping, while the rate of non-neglect specific errors was $22 \%$. Contrarily, during the resection of the rear pole of the tumor, number of neglect specific errors increased significantly $(+46 \%)$, while the number of non-
Line bisection test-postoperative phase. d Bells test-preoperative phase. e-g Bells test-postoperative phase

neglect specific errors changed slightly $(+3 \%)$. However, this location was further away from the area identified with nTMS as susceptible to neglect (inferior parietal lobule). Due to the transient manner of errors during the stimulation of this area, the resection was carried on. Using the acquired information, maximal safety was achieved during the resection. Postoperative MRI scan at $24 \mathrm{~h}$ confirmed total resection of the tumor (Fig. 3).

\section{Postoperative phase}

\section{MR imaging $24 \mathrm{~h}$ postoperatively}

MRI showed postoperative changes with T1 hyperintensivity on the surface of the resection site as a sign of accumulated blood. FLAIR showed intensity of about $16 \times 6 \mathrm{~mm}$ in size in the area constructing the lateral wall of the posterior horn the right lateral ventricle. Presence of some postoperative air in front of the frontal poles was described.

\section{Neuropsychology}

Twenty-four hours after the surgery a moderate ego- and allocentric visual neglect has been detected peripersonally, for the left side of the space, which was clearly observed in the behavior of patient (e.g., the bisection of horizontal lines were happened from right side to the left side) and also in the recorded tests (an obvious, average orientation of $2.5 \mathrm{~cm}$ for right side in the line-bisection test, and weaker performance with $40 \%$ in the 
Bells test due to the omission of the left elements). The patient lies on the right side of the bed, despite the fact that the surgical scar was also located on the right side of her head. Nevertheless, personal and/or extrapersonal neglect syndrome was not perceived by the clinical observation.

On the fourth day following the operation (more than $72 \mathrm{~h}$ later), visuospatial capabilities show a significant improvement (number of identified elements in the left visual filed was increased with $17 \%$ in the Bells test); however, vision problems are still indicated by the patient ("Something is weird"); therefore, a confrontational visual field exam was completed based on the Dean-Woodcock Neuropsychological Battery. Complete right and left side, as well as simultaneous (concomitant, extinction) testing of the upper, middle, and lower visual fields shows partial left homonymous hemianopia in the patient (total left: point $=0$, " $w$ " value $=415 /$ severe; , total simultaneous: point $=$ 0 , “ $w$ " value $=429 /$ severe ; total right: point $=24, " w$ " value $=$ 488/normal zone).

It is of note that on the basis of surgical planning based on preoperative assessment of optic radiation as well as postop MR images such partial visual field defect could well have been anticipated upon gross total tumor resection.

On the fifth day, the homonymous hemianopia was still detected, while the symptoms (and behavior) referred to the neglect completely disappeared. The Bells test has already performed correctly, with similar search strategy as before the surgery, and no significant difference was observed between the length of horizontal lines that have been halved in the line-bisection task.

Regarding the other neuropsychological functions, the preand postoperative results were summarized in Table 2 . The alteration in visuospatial attention functions was illustrated in Fig. 4.

\section{Conclusion}

In this work, we have identified a complex pre-, intra-, and postoperative protocol for the assessment of neglect in conjunction with awake surgery upon gross total removal of a low-grade glioma. Navigated-MRI-based-transcortical magnetic stimulation has been included as integrated part of the preoperative workup and planning.

According to the pre- and intraoperative assessments and the results presented in this case, the applied paradigm was useful for identifying eloquent areas of visuospatial attention and had predictive value for the development of postoperative neglect-like symptoms. In addition to neuromonitoring, this was further improved by the surgical strategy followed in the intraoperative. Our case report supports the literature data that lesions of the superior temporal gyrus play less role in the appearance of permanent neglect-like symptoms, but rather can result in transient changes. The paradigms established here should aid supramaximal safe resection of tumors aiding prolonged survival and better quality of life of our patients.

Supplementary Information The online version contains supplementary material available at https://doi.org/10.1007/s00701-021-04822-2.

Funding Open access funding provided by University of Pécs. This study was supported by the Thematic Excellence Program 2020 (2020-4.1.1.TKP2020)-Institutional Excellence Sub-program of the Ministry for Innovation and Technology in Hungary, within the framework of the 5 thematic program of the Pécs University. The study was also financed by EFOP-3.6.2.-16-2017-00008 "The role of neuro-inflammation in neurodegeneration: from molecules to clinics", GINOP-2.3.2-15-2016-00048, GINOP-2.2.1-15-2017-00067 "Networked Analytical Opportunities and Data Utilization in Healthcare" and the Hungarian Brain Research Program 2.0 Grant No. 2017-1.2.1-NKP-2017-00002 as well as the National Research Development and Innovation Office-Grant No. OTKA K-134555.

\section{Declarations}

Ethics approval and consent to participate All procedures performed in this study involving human participant were in accordance with the ethical standards of the institutional and/or national research committee and with the 1964 Helsinki Declaration and its later amendments or comparable ethical standards. For this type of study, formal consent is not required.

Open Access This article is licensed under a Creative Commons Attribution 4.0 International License, which permits use, sharing, adaptation, distribution and reproduction in any medium or format, as long as you give appropriate credit to the original author(s) and the source, provide a link to the Creative Commons licence, and indicate if changes were made. The images or other third party material in this article are included in the article's Creative Commons licence, unless indicated otherwise in a credit line to the material. If material is not included in the article's Creative Commons licence and your intended use is not permitted by statutory regulation or exceeds the permitted use, you will need to obtain permission directly from the copyright holder. To view a copy of this licence, visit http://creativecommons.org/licenses/by/4.0/.

\section{References}

1. Bonato M (2012) Neglect and extinction depend greatly on task demands: a review. Front Hum Neurosci 6:195

2. Giglhuber K, Maurer S, Zimmer C, Meyer B, Krieg SM (2017) Evoking visual neglect-like deficits in healthy volunteers-an investigation by repetitive navigated transcranial magnetic stimulation. Brain Imaging Behav 11:17-29

3. Harvey M, Milner AD, Roberts RC (1995) An investigation of hemispatial neglect using the landmark task. Brain Cogn 27:59-78

4. Herbet G, Duffau H (2019) Awake craniotomy and bedside cognitive mapping in neurosurgery. In: Neurosurgical Neuropsychology. Elsevier, pp 113-138 
5. Krieg SM, Lioumis P, Mäkelä JP, Wilenius J, Karhu J, Hannula H, Savolainen P, Lucas CW, Seidel K, Laakso A, Islam M, Vaalto S, Lehtinen H, Vitikainen AM, Tarapore PE, Picht T (2017) Protocol for motor and language mapping by navigated TMS in patients and healthy volunteers; workshop report. Acta Neurochir 159:11871195. https://doi.org/10.1007/s00701-017-3187-z

6. Li K, Malhotra PA (2015) Spatial neglect. Pract Neurol 15:333-339. https://doi.org/10.1136/practneurol-2015-001115
7. Mort DJ, Malhotra P, Mannan SK, Rorden C, Pambakian A, Kennard C, Husain M (2003) The anatomy of visual neglect. Brain 126:1986-1997

8. Vallar G, Perani D (1986) The anatomy of unilateral neglect after right-hemisphere stroke lesions. A clinical/CT-scan correlation study in man. Neuropsychologia 24:609-622

Publisher's note Springer Nature remains neutral with regard to jurisdictional claims in published maps and institutional affiliations. 